\title{
Contexte socio-économique et performances scolaires en Afrique de l'Ouest
}

\section{Touré Krouélé}

\section{OpenEdition}

\section{Journals}

Édition électronique

URL : https://journals.openedition.org/ries/6333

DOI : $10.4000 /$ ries.6333

ISSN : 2261-4265

Éditeur

France Education international

Édition imprimée

Date de publication : 1 septembre 2018

Pagination : 23-27

ISBN : 978-2-85420-619-7

ISSN : $1254-4590$

Référence électronique

Touré Krouélé, "Contexte socio-économique et performances scolaires en Afrique de l'Ouest », Revue internationale d'éducation de Sèvres [En ligne], 78 | septembre 2018, mis en ligne le 01 septembre 2020 consulté le 25 juin 2021. URL : http://journals.openedition.org/ries/6333 ; DOI : https://doi.org/ $10.4000 /$ ries. 6333

Ce document a été généré automatiquement le 25 juin 2021.

(C) Tous droits réservés 


\title{
Contexte socio-économique et performances scolaires en Afrique de l'Ouest
}

\author{
Touré Krouélé
}

1 Les systèmes éducatifs en Afrique de l'Ouest présentent des similitudes mais également de profondes différences. Dans cette partie du monde, les États ont été colonisés et, dès les Indépendances, ils ont poursuivi le développement de l'éducation formelle selon le modèle de la puissance colonisatrice. Des États francophones comme le Bénin, le Burkina-Faso, la Côte d'Ivoire, le Mali, le Niger, le Sénégal et le Togo ont en commun l'usage du français comme langue officielle et du franc CFA comme monnaie unique. Tous situés au sud du Sahara, en Afrique occidentale, ils partagent également la même histoire coloniale et scolaire. Après les Indépendances dans les années 1960, ces États s'efforcent d'adapter les programmes scolaires hérités de la colonisation aux réalités locales, tout en restant dans le contexte francophone.

Dans les années 1980, des institutions internationales comme la Banque mondiale, la Banque africaine de développement (BAD), le Programme des Nations Unies pour le développement (PNUD) ou l'Unesco produisent des rapports où elles montrent la nécessité de valoriser les ressources humaines par le développement de l'éducation et de la formation. Le PNUD s'appuie alors sur l'Indice de développement humain (IDH) comme outil de travail pour évaluer le niveau de développement des pays. Cet indice se calcule à partir de trois indicateurs : la richesse du pays (à travers son produit intérieur brut), l'espérance de vie de la population à la naissance et les conditions globales d'éducation (taux d'alphabétisation des adultes de plus de 15 ans, taux brut de scolarisation au primaire, secondaire et supérieur, puis durée moyenne de la scolarité). La valeur de l'IDH pour un pays traduit son niveau de développement humain. À partir du calcul de cet indice, le PNUD procède chaque année à un classement mondial. C'est ainsi qu'en 2014, le Bénin est classé $166^{\mathrm{e}}$ sur 188 États, avec un IDH de 0,480. Le Sénégal, avec un IDH de 0,466 est $170^{\mathrm{e}}$ quand la Côte d'Ivoire occupe la $172^{\mathrm{e}}$ place, avec un IDH égal à 0,462 . Les rangs occupés par ces États traduisent un retard important en matière 
de développement humain. Cette situation interroge en premier lieu l'évolution de l'éducation dans ces pays.

3 La mission principale des systèmes éducatifs est d'éduquer la population pour produire le capital humain capable de contribuer au développement de tous les secteurs d'activité du pays. Mais quel est le contexte économique dans lequel fonctionnent les systèmes éducatifs en Afrique occidentale francophone? Les apprenants réalisent-ils des performances identiques d'un pays à l'autre?

Pour répondre à ces questions, notre recherche s'est donné pour objectif d'analyser le lien entre le contexte socio-économique des sept États retenus et les performances des apprenants en termes d'acquisition de connaissances. L'hypothèse testée a été la suivante: «Il existe un lien entre les performances des apprenants et le niveau de pauvreté du pays, le niveau de qualification des enseignants, et la part du PIB consacrée à l'éducation ». La méthode utilisée pour vérifier cette hypothèse a consisté à analyser les données issues de documents et rapports produits par de organisations internationales indépendantes comme la BAD, l'Unesco, le PNUD et la Confemen ${ }^{1}$. Une analyse statistique des variables pertinentes pour cette investigation a été privilégiée.

\section{Résultats}

5 Le Bénin, le Burkina-Faso, la Côte d'Ivoire, le Mali, le Niger, le Sénégal et le Togo ont traversé, entre 1980 et 2012, des périodes marquées par des crises socio-économiques justifiant un retard dans le domaine de l'éducation. Les structures financières internationales sont intervenues pour imposer des programmes d'ajustement structurel, dont les effets sur la vie des populations ne sont guère encourageants. Très endettés, ces États doivent faire face au service de la dette extérieure, dont le poids sur les budgets nationaux est trop lourd. Ils consacrent toutefois une partie de leurs ressources à la construction d'établissements scolaires et à la formation des enseignants. Ces ressources financières investies relèvent en grande partie du budget national consacré à l'éducation. L'assistance ou l'aide publique au développement (APD) intervient également dans les dépenses publiques d'éducation (Bayemi, 2014). Dans un contexte où les taux de croissance de la population de ces pays sont parmi les plus élevés au monde (entre $2,4 \%$ et $4 \%^{2}$ ), les populations scolarisable et scolarisée connaissent une forte croissance. En conséquence, la taille des classes est généralement très importante, surtout en milieu urbain. Les enseignants recrutés pour encadrer ces classes ne sont pas toujours formés.

6 Les recommandations des organisations internationales en matière d'éducation consistent à inciter les pays pauvres à combattre la pauvreté et l'analphabétisme en scolarisant tous les enfants en âge scolaire, de manière qu'ils achèvent tout le cycle d'enseignement primaire. Une évaluation des apprenants dans les matières essentielles comme la maîtrise de la langue et celle des mathématiques est nécessaire pour déterminer la performance des systèmes éducatifs. L'évaluation réalisée en 2014 par le PASEC dans dix pays d'Afrique au sud du Sahara peut être un outil d'analyse du lien entre qualité des ressources investies et performances des apprenants.

$7 \quad$ Les données présentées plus bas montrent une absence de lien clair entre le nombre de croissances positives du PIB (ou diminution de la pauvreté) et les trois variables que sont les pourcentages d'enseignants qualifiés, la part du PIB consacrée à l'éducation et les résultats du PASEC 2014. De même il n'y a pas de lien entre la part du PIB consacrée 
à l'éducation et les deux variables que sont le pourcentage d'enseignants qualifiés et les résultats du PASEC 2014. Il n'y a pas non plus de lien entre le pourcentage d'enseignants qualifiés et les résultats du PASEC 2014.

Des inégalités entre les sept pays apparaissent dans la progression du PIB. Ainsi, le Bénin et le Burkina Faso ont une progression du PIB identique, mais diffèrent fortement dans les résultats au PASEC 2014. Selon le tableau, l'explication provient de la différence dans le pourcentage d'enseignants qualifiés, car le Burkina dispose de deux fois plus d'enseignants qualifiés que le Bénin. Le niveau de qualification des enseignants n'est donc pas une variable négligeable dans les performances des apprenants.

Tableau $\mathrm{n}^{\circ} 1$. Variables liées aux compétences des élèves suivant la situation économique des États

\begin{tabular}{|c|c|c|c|c|c|c|c|}
\hline \multirow{2}{*}{ Pays } & \multirow{2}{*}{$\begin{array}{l}\text { Progression du } \\
\text { PIB entre } 1980 \\
\text { et } 2012\end{array}$} & \multirow{2}{*}{$\begin{array}{l}\% \\
\text { d'enseignants } \\
\text { qualifiés }\end{array}$} & \multirow{2}{*}{$\begin{array}{lr}\text { Part du } & \text { PIB } \\
\text { consacrée } & \text { à } \\
\text { l'éducation } & \end{array}$} & \multicolumn{4}{|c|}{$\begin{array}{l}\text { PASEC } 2014 \text { : \% d'élèves ayant passé } \\
\text { le seuil de maîtrise minimale }\end{array}$} \\
\hline & & & & $\begin{array}{l}\text { Langue } \\
2^{\circ} \mathrm{P}^{3}\end{array}$ & $\begin{array}{l}\text { Maths } \\
2^{\circ} \mathrm{P}\end{array}$ & $\begin{array}{l}\text { Langue } \\
5^{\circ} \mathrm{P}^{4}\end{array}$ & $\begin{array}{l}\text { Maths } \\
5^{\circ} \mathrm{P}\end{array}$ \\
\hline Benin & 4 & 43 & 5,0 & 6,3 & 33,5 & 51,7 & 39,8 \\
\hline $\begin{array}{l}\text { Burkina } \\
\text { Faso }\end{array}$ & 4 & 86 & 4,3 & 35,4 & 59,2 & 56,9 & 58,8 \\
\hline $\begin{array}{l}\text { Côte } \\
\text { d'Ivoire }\end{array}$ & 0 & 100 & 4,4 & 11,7 & 33,8 & 48,0 & 26,9 \\
\hline Mali & 2 & 50 & 4,2 & \multicolumn{4}{|c|}{ Pas d'évaluation PASEC 2014} \\
\hline Niger & 1 & 96 & 4,5 & 6,4 & 27,8 & 6,4 & 7,6 \\
\hline Sénégal & 3 & 48 & 5,6 & 28,9 & 60,3 & 61,1 & 58,8 \\
\hline Togo & 1 & 77 & 4,4 & 20,1 & 41,3 & 38,4 & 47,6 \\
\hline
\end{tabular}

Cependant le cas de la Côte d'Ivoire, qui part d'une croissance du PIB en dessous de zéro et réalise $100 \%$ d'enseignants qualifiés mais obtient des résultats au PASEC 2014 faibles en langue $2^{\circ} \mathrm{P}$, montre que le "niveau de qualification des enseignants » n'est pas la seule variable explicative des performances des élèves. De son côté, le Niger dispose de 96 \% d'enseignants qualifiés, mais occupe les dernières places au PASEC 2014. Cela montre une fois encore que le niveau de qualification des enseignants ne suffit pas pour qu'un pays améliore ses performances scolaires.

10 Le Sénégal et le Burkina Faso réalisent, à la même évaluation du PASEC, les meilleurs scores dans trois matières sur les quatre retenues. Ils sont en tête par rapport aux dix pays évalués mais présentent de profondes divergences au niveau des trois autres variables que sont la progression du PIB par habitant, le pourcentage d'enseignants qualifiés et la part du PIB éducation. Si le Burkina Faso dispose d'un PIB par habitant et d'un pourcentage d'enseignants qualifiés plus importants, sa part du PIB éducation 
reste faible, comparée à celle du Sénégal qui demeure la plus élevée dans la sousrégion. Le profil de ces deux pays autorise à retenir que les performances des apprenants au PASEC 2014 ont un lien fort avec « le niveau de pauvreté du pays », « le niveau de qualification des enseignants » et « la part du PIB consacrée à l'éducation ». La combinaison de ces trois variables est donc nécessaire pour comprendre les performances en éducation des pays évalués.

11 En bref, les résultats de cette étude montrent qu'aucun pays retenu ne fournit des indicateurs suffisamment excellents pour justifier des résultats toujours performants. C'est du reste pourquoi aucun pays n'obtient la moyenne dans les quatre disciplines retenues à l'évaluation PASEC. Si le Togo, le Bénin et le Sénégal semblent avoir réalisés une meilleure démocratisation de leur système éducatif respectif, le problème de la qualité des enseignements continue à se poser car les enseignants recrutés ne sont pas toujours qualifiés. D'ailleurs, tous ces pays ont besoin de l'aide publique au développement pour le financement complet des systèmes éducatifs. Enfin, que retenir des analyses effectuées?

12 Cette étude met d'abord en évidence des différences à plusieurs niveaux concernant les sept pays pris en compte. La situation économique des États (à travers le PIB par habitant et la part du PIB consacrée à l'éducation), les ressources financières, matérielles, pédagogiques et humaines investies dans l'éducation et les performances scolaires enregistrées par chaque pays à l'évaluation du PASEC 2014. Ces différences sont d'autant plus réelles que chaque pays est confronté à des problèmes spécifiques. Géographiquement, si le Sénégal, la Côte d'Ivoire, le Togo, le Bénin sont des pays côtiers, le Mali, le Burkina Faso, le Niger sont des pays sahéliens. Ces pays n'ont pas les mêmes superficies. Leurs économies n'ont pas la même diversité ni le même dynamisme. Le taux de croissance de la population diffère d'un pays à l'autre. Et les inégalités entre "les élites politico-administratives ", qui représentent "une minorité parasitaire » et la majorité de la population continuent de se creuser, favorisant la marginalisation, l'analphabétisme et la paupérisation des plus faibles (Bayemi, 2014).

13 Le second enseignement est qu'en dépit des différences, ces pays sont confrontés aux mêmes problèmes scolaires. La croissance de la population jeune entraîne une croissance globale de la scolarisation. Chaque État s'est donné pour objectif de faire progresser la couverture scolaire. Et à ce titre, les taux de scolarisation s'améliorent d'année en année, dans chaque pays. La lutte contre la déscolarisation se poursuit, la prise de conscience de l'équité et de l'inclusion scolaire est de mieux en mieux partagées. Ces sept systèmes éducatifs dépendent en partie de l'aide publique au développement. Les politiques éducatives nationales s'inscrivent dans le cadre des orientations internationales définies d'une part par les Objectifs du millénaire pour le développement (OMD) entre 2000 et 2015 et d'autre part, par les Objectifs de développement durable (ODD) en cours entre 2016 et 2030.Au titre notamment des OMD, chaque État s'est engagé dans l'adoption d'une loi sur l'obligation scolaire.

14 Cette comparaison portant sur un échantillon de sept pays africains en développement relève que l'efficacité interne des systèmes éducatifs dépend à la fois du niveau de pauvreté du pays, du niveau de qualification des enseignants et de la part du PIB consacrée à l'éducation. En fait, aucun des trois indicateurs n'a pu, à lui seul, garantir la performance d'un système éducatif. Cette performance est donc produite par un agrégat de facteurs qui interagissent. Ainsi, le «niveau de pauvreté du pays » peut interagir avec le «niveau de qualification des enseignants» et «la part du PIB 
consacrée à l'éducation ». Mais l'étude montre qu'il n'existe pas de déterminisme dans ces interactions. Au-delà des variables manipulées, il faut tenir compte d'un autre facteur dont l'influence est inévitable sur les politiques éducatives dans des pays en développement: la «volonté politique». Celle-ci renvoie à la question de la gouvernance éducative. En fait, les performances d'un système éducatif traduisent la nature de la politique éducative appliquée. C'est donc aussi une question de gouvernance en matière éducative. Les richesses dont dispose un État ne s'orientent pas seules dans les différents secteurs clés de l'économie, du développement et notamment de l'éducation. Il faut une volonté politique consciente de l'importance de l'éducation pour le développement du pays. Est-il besoin de rappeler ici qu'un chef d'État africain soutenait, au début des Indépendances, que «l'éducation est la cinquième roue du carrosse »? Outre l'absence de prise de conscience, il faut noter que la corruption des élites au pouvoir ne garantit pas toujours une gestion efficiente des ressources nationales. Aussi, pour rendre les systèmes éducatifs africains performants, l'application d'une bonne gouvernance est nécessaire. Et pour cela, le recadrage des politiques éducatives nationales à l'aide des instruments d'accompagnement mis en place par la communauté internationale (OMD, ODD) ainsi que les conditionnalités de l'aide publique au développement ne sont pas négligeables. $\mathrm{n}$

\section{BIBLIOGRAPHIE}

BAD/PNUD/OCDE (2016) : Perspectives économiques en Afrique (Annexe statistique). En ligne [DOI : http://dx.doi.org/10.1787/aeo-2016-fr].

BAD (2016) : Rapport sur le développement en Afrique 2015. Croissance, pauvreté et inégalités : lever les obstacles au développement durable. En ligne [http://bit.ly/2mSdzU7]

BAYEMI G. (2014) : Financement public de l'éducation en Afrique. Volume global, destination et marge d'amélioration de l'efficience, Aix-en-Provence, Persée.

CONFEMEN (2014) : PASEC 2014 performances des systèmes éducatifs en Afrique subsaharienne francophone. Compétences et facteurs de réussite au primaire. En ligne [http://bit.ly/2M5Te8Z].

KOUASSI B. (éd.) (2008) : Pauvreté des ménages et accès à l'éducation en Afrique de l'Ouest. Burkina Faso, Côte d'Ivoire, Ghana et Togo, Paris, Karthala.

PNUD (2016) : Rapport sur le développement humain 2016. Le développement humain pour tous. En ligne [http://bit.ly/2veH3PO].

\section{NOTES}

1. Conférence des ministres de l'éducation des États et Gouvernements de la Francophonie.

2. PNUD, Rapport sur le développement humain 2016, p. 250-251: entre 2010 et 2015, les taux de croissance démographique annuelle moyenne sont les suivants: Côte d'Ivoire $(2,4)$; Sénégal $(3,1)$; Togo $(2,7)$; Bénin $(2,7)$; Burkina Faso $(2,9)$; Mali $(3,0)$; Niger $(4,0)$. 
3. Deuxième année du primaire (le cycle primaire dure six années)

4. Cinquième année du primaire.

\section{INDEX}

Index géographique : Afrique de l'Ouest, Afrique francophone

Mots-clés : résultats d'apprentissage, financement de l'éducation, PASEC : programme d'analyse des systèmes éducatifs

Keywords : learning outcomes, educational finance, PASEC: Programme for the Analysis of the Educational Systems

Palabras claves : resultados de aprendizaje, financiación de la educación, PASEC: programa de análisis de los sistemas educativos

\section{AUTEUR}

\section{TOURÉ KROUÉLÉ}

Touré Krouélé, titulaire d'un CAPES de philosophie, a enseigné en lycée pendant dix ans. Docteur en sociologie de l'éducation, il enseigne cette discipline à l'École normale supérieure d'Abidjan (Côte d'Ivoire) depuis 2003. Ses recherches portent principalement sur les questions d'échec scolaire, d'inégalité d'accès, de parcours et de réussite des élèves. Il s'intéresse également au métier d'élève ou d'enseignant ainsi qu'aux politiques scolaires. Courriel : tk_krouele@yahoo.fr 